\title{
Reflexión sobre la publicación académica y el acceso abierto a partir de la experiencia de Redalyc
}

Thoughts on academic publishing and Open Access regarding the experience of Redalyc

\author{
Eduardo Aguado-López \\ Universidad Autónoma del Estado de México, México \\ eduardo.aguado@redalyc.org \\ Arianna Becerril-García \\ Universidad Autónoma del Estado de México, México \\ arianna.becerril@redalyc.org \\ Salvador Chávez-Ávila \\ Universidad Autónoma del Estado de México, México \\ salvador.chavez@redalyc.org
}

\section{ReSUMEN:}

El presente trabajo conjuga una serie de reflexiones acerca de los retos y avances de la publicación científica en acceso abierto a través de los últimos dieciséis años, en Latinoamérica. Con el punto de vista de su experiencia en el desarrollo conceptual y tecnológico de Redalyc, los autores comparten propuestas para construir una plataforma para la publicación académica latinoamericana de las Ciencias Sociales y las Humanidades principalmente, de acceso abierto no comercial, colaborativo, sustentable y protegido. Además, hacen hincapié en cuatro temas centrales: el modelo de publicación de Latinoamérica, los sistemas de evaluación, la era digital y la comunicación académica, y el acceso abierto y la sustentabilidad.

Palabras clave: Publicaciones académicas, Acceso Abierto, Sustentabilidad, Tecnologías, Publicaciones digitales, AmeliCA, América Latina y el Caribe .

\section{Abstract:}

The present work presents a series of reflexions regarding the challenges and advances in scientific publications in Open Access, throughout the last sixteen years, in Latin America. With a point of view based on the experience of the conceptual and technological development in Redalyc, the authors share their proposals to build a platform for the latin american academic publishing in Social Sciences and Humanities, mainly in non commercial, collaborative, sustainable and protected Open Access. They also emphasize four relevant subjects: Latin America's publishing model, evaluations systems, the digital era and the academic communication, and Open Access and sustainability.

KeYWORDs: Academic Publications, Open Access, Sustainability, Technologies, Digital Publications , AmeliCA , Latin America and the Caribbean .

\section{INTRODUCCIÓN ${ }^{1}$}

El presente trabajo busca ser un ensayo reflexivo basado en la experiencia de sus autores en más de una década y media de participar conceptual y tecnológicamente con propuestas para construir una plataforma para la publicación académica latinoamericana de las Ciencias Sociales y las Humanidades principalmente, de acceso abierto no comercial, colaborativo, sustentable y protegido.

Los autores son académicos fundadores y directores del Sistema de Información Científica Redalyc (ht tps://www.redalyc.org) de la Universidad Autónoma del Estado de México, México, y las reflexiones son producto de esa práctica crítica, reflexiva y comprometida.

Recepción: 19 de febrero de 2019 | Aceptación: 13 de marzo de 2019 | Publicación: 25 de abril de 2019 
El trabajo no tiene la estructura de un artículo científico. En cada párrafo podrían citarse decenas de textos, pero lo más alejado es pretender mostrar erudición. De modo que este trabajo debe leerse como reflexiones de tres actores que han dedicado casi dos décadas al desarrollo de tecnología bajo la filosofía de acceso abierto.

\section{El Modelo de PUblicación del Sur}

Sin embargo, se muestran diversos puntos de tensión:

- Hay una gran diversidad de posiciones sobre cómo avanzar, difícilmente consensuables.

- Hay una brecha tecnológica que se amplía día a día, entre países, instituciones y revistas.

- Los caminos a seguir por los sistemas regionales son inciertos, y tendrán cambios importantes.

- La región carece de estrategias de acceso abiertoregionales y globales consensuadas; los actores sólo se han sumado a las iniciativas existentes y las han legitimado.

- Muchas veces se asume como adecuado el modelo del Norte basado en el cobro de tasas de publicación (Article Processing Charges-APC), que tendría consecuencias funestas para la publicación académica abierta y pública. Los APC no son práctica común en la región y se estiman inviables como estrategia o vía de inserción del Sur, incluida ALyC, en la conversación global. En este contexto es importante -aunque limitada- la Declaración de la Primera Reunión de Consorcios de Iberoamérica y el Caribe (2017), que se opone al cobro de tasas de publicación (APC), así como las diferencias marcadas entre el Plan S y AmeliCA (2019). ${ }^{2}$

- En América Latina y el Caribe (ALyC) se asume como modelo, en general, que el acceso abierto (AA) es colaborativo, no comercial, inclusivo y que camina hacia la sustentabilidad, principios que discrepan y se oponen a la visión original de las 3 "B" (Budapest, Berlín y Bethesda), en particular a la idea de compartir sin restricciones y que se permita la comercialización y reutilización irrestricta. En la región latinoamericana hay múltiples voces que consideran que si es financiado con fondos públicos no se debe permitir el lucro, posición que se contradice con las licencias CC-BY propuestas por las tres "B". A este respecto, Latindex-Redalyc-CLACSO e IBICT emitieron su posición con la Declaración de México (2017) ${ }^{3}$, en la cual recomiendan usar CC BY-NC-SA: Atribución - No Comercial - Compartir Igual - No Derivadas.

- Hay severas contradicciones y discrepancias entre las políticas impulsadas para el fortalecimiento de la ciencia, la internacionalización y el AA por parte de los actores centrales que determinan la investigación: consejos de ciencia tecnología, universidades, centros de investigación y organismos de financiación.

- La valoración de los científicos y la ciencia publicada está basada, cada vez más, en indicadores de bases de datos del llamado mainstream que en realidad refieren a dos bases de datos comerciales: WoS y Scopus. La segunda, pertenece a Elsevier, el principal editor con más de 2.500 revistas y el "juez" que determina la "calidad", "posicionamiento" e "impacto" a través de los indicadores de Scopus.

- Las tradicionales formas de comunicación de las diversas disciplinas (libro, ensayo, artículo) han cedido terreno a un "único" formato válido, el paper: objetivo, método, resultados, discusión y conclusiones; estructura difícil para la mayor parte de la investigación de corte no empírico en las Ciencias Sociales y Humanas.

- La falta de estrategias de sustentabilidad y de recursos del modelo de ciencia abierta y pública, en contraste con el aumento sostenido de recursos para la compra de bases de datos y publicaciones electrónicas.

- Entre las tensiones principales se encuentran los parámetros adoptados en años recientes por los sistemas de evaluación en los diferentes órdenes de la actividad académica. Prácticamente sin excepción, en los últimos años dichos parámetros se fundan en las bases comerciales WoS y Scopus. 
- La reconfiguración del movimiento de AA a nivel mundial, con nuevos modelos de negocio, que genera nuevas exclusiones como respuesta de las editoriales comerciales, solapadas por la falta de firmeza por parte de los editores en la definición de políticas de AA y por el bajo reclamo de las revistas de calidad para ocupar su lugar en los sistemas de evaluación científica.

Dos hechos que esbozan un futuro incierto y sinuoso son:

- El control y legitimación de la publicación académica y sus derivados en manos de los monopolios editoriales privados.

- La inexistente propuesta a nivel regional, nacional, e incluso institucional, de generar espacios, tecnologías, políticas, etc., que permitan retomar el control de la publicación académica y científica a la comunidad académica.

Todo ello constituye un panorama incierto, difícil y de mayor complejidad a 15 años de la Declaración de Budapest (BOAI), aunque se cuente con leyes, mandatos y repositorios para la comunicación académica abierta y pública.

\section{LA PUBLICACIÓN ACADÉMICA Y LOS SISTEMAS DE EVALUACIÓN}

La publicación académica ha tenido drásticos cambios que obedecen a dos procesos: el surgimiento de internet y la digitalización de los formatos de publicación ( $\mathrm{y}$, en consecuencia, de la comunicación y evaluación de la ciencia). Se transformaron los sistemas de participación, reconocimiento, transmisión, etc., y con ello el sistema de evaluación. Entre las principales transformaciones pueden mencionarse:

A) La globalización hizo que los modelos predominantes se impusieran, se generalizaran y se consideraran correctos, adecuados y comunes a todos. Primero fue la internacionalización y con ella los indicadores, los sistemas de difusión y bases de datos (ISI); no importaron los contextos, las condiciones del sistema, las necesidades locales, nacionales y regionales. El medio se convirtió en fin. El Sur, de repente, se encontró con que había revistas globales que en su mayoría desconocía y por ende consultaba muy poco -sólo una micro élite participaba de y en ellas-, pero ahora debía leerlas y, principalmente, publicar en esas revistas para participar en el diálogo mundial de la ciencia. Esto tuvo lugar sin distinción de disciplinas e impuso como condición un pago por el acceso.

B) A la legitimación de las bases, le siguió la legitimación de diversos indicadores bibliométricos: Factor de Impacto, SCimago Journal Rank e índice H (FI-SJR-H), todos ellos basados en citas.

C) Se concluyó, de una u otra forma, que sólo tenía valor y era importante lo que estaba en las bases llamadas el "mainstream" de la ciencia.

D) El cambio más drástico, con consecuencias aún no visualizadas y analizadas, es que los productos de la investigación dejaron de ser significativos por sus características propias (objetivos, calidad, vinculación con las necesidades nacionales y locales, etc.), las características esenciales de los procesos dejaron de ser importantes -en algunos países y universidades centrales se llegó al caso de ni siquiera registrarlas o mencionarlas-, sólo importaban si tenía presencia en el mainstream (WoS-Scopus), su lugar en el núcleo 'central' a partir del FI-SJR-H y las citas recibidas.

E) Los índices nacionales de revistas cambiaron sus parámetros ajustándose los del mainstream e integraron y asignaron valores o posiciones a las revistas a partir de si estaban o no allí, lo que tuvo como consecuencia que en algunos países cientos de revistas salieran del índice nacional.

F) Los sistemas regionales y experiencias institucionales no tuvieron valor e importancia en los procesos de evaluación. 
G) La mayor paradoja pudo observarse cuando las revistas no fueron consideradas de calidad, a menos que estuvieran incluidas en WoS o Scopus. Las revistas mantenían sus procesos editoriales, sus tasas de rechazo y su posicionamiento internacional; conservaban las mismas características, pero sólo se reconocieron de calidad cuando fueron incluidas en el mainstream.

H) Los cambios llegaron incluso a la hoja de vida de los académicos. Era posible registrar todas sus actividades, pero tenía mayor peso, o sólo se tomaba en cuenta, lo que estaba en el mainstream.

I) Entre los académicos y editores se inició una forma de disonancia cognitiva: por un lado, criticaban el proceso de evaluación; por otro, sentían que su trabajo o su revista era de mayor valor y más calidad si tenían un importante indicador bibliométrico, o si tenía más citas que otro colega u otra revista; es decir, bajo las reglas que el mainstream definía.

J) Las universidades no quedaron exentas. Los rankings y las oficinas de posicionamiento se convirtieron en prácticas y oficinas con la tarea de dar seguimiento al posicionamiento alcanzado por la institución; no tardaron mucho en identificar que, si modificaban los parámetros de inserción, financiamiento, promoción, etc., de la investigación, mejorarían posiciones: el fin era la posición, el lugar en el ranking. Nuevamente, el medio se convirtió en fin.

K) Los monopolios editoriales empezaron a comprar y controlar el circuito de la ciencia, las redes de científicos, los altmetrics, los sistemas de eprints, etc., con el fin de trasladar el modelo de evaluación del mainstream a los procesos de acceso abierto: los repositorios y los sistemas de gestión de la investigación (CRIS por sus siglas en ingles).

Enfrentamos un momento difícil y complejo, que nos permite afirmar, casi sin duda, que con el presente modelo de evaluación, el acceso abierto no comercial no tiene futuro.

\section{LA COMUNICACIÓN ACADÉMICA EN LA ERA DIGITAL}

El gran reto de la publicación académica es la transición a lo digital. El primer paso ha sido la transición al soporte electrónico. Sin embargo, el reto es transformarse en publicaciones digitales.

La mayoría de las revistas regionales continúan sustentando sus decisiones centrales a partir de la revista impresa, aun cuando nacen como revistas electrónicas y el producto lo trasladan a un soporte electrónico (PDF); el reto a enfrentar es que la revista opere bajo la lógica de un proceso digital. Una publicación científica digital se conceptualiza y se gesta en un ecosistema digital, es diseñada para desarrollarse en un medio tecnológico y para usuarios de textos científicos digitales. Este proceso se considera desde la entrada de información, así como todos los procesos de gestión, comunicación, edición, difusión y despliegue de la información, lo que incluye la salida de los formatos de lectura, interoperabilidad, discoverabilidad y generación de métricas.

El proceso editorial exige: i) separar los procesos propios de lo impreso y lo electrónico o digital; ii) subordinar lo impreso a lo digital (XML).

Transitar al formato XML-JATS, base de una revista digital, permite: i) separar el contenido de los textos científicos de cualquier noción de formato o salida; ii) al separar el contenido, preservarlo, ya que es independiente al formato o plataforma de visualización; iii) generar diversos formatos de lectura derivados del marcaje XML-JATS: visores de lectura, PDF, HTML, ePub, etc; iv) mejorar la indexación de contenidos hipertextuales por los motores de búsqueda, incrementando su visibilidad, acceso e impacto; v) incorporar nuevos formatos de acceso a la información como multimedia, audio, video, hipertexto, cartografía, entre otros, con el fin de enriquecer la experiencia del lector; vi) novedosos formatos de visualización: gifs, gráficas dinámicas, manipulación de gráficas y procesos en la web, fotografías con enlaces a imágenes de alta calidad (vitales por ejemplo en la medicina, en la astronomía y en el arte) de forma que nos permitan apreciar los cuadros dentro del cuadro, las pinceladas, los grosores de los materiales, las texturas, etc.; vii) 
hipervínculos que permiten conexiones, por ejemplo para conocer la referencia -al pasar el ratón-y dirigirse a la fuente misma, permitiendo un texto interactivo (esto no se refiere exclusivamente a la cita, sino a múltiples elementos que pueden ser enlazados y que no constituyen una cita en sí misma); viii) cumplir con el estándar de la W3C que es el XML, así como con el estándar ANSI/NISO Journal Article Tag Suite, además de cubrir los criterios de los sistemas de información regionales y globales en este aspecto; ix) incluir los datos, la materia prima de la investigación, factores esenciales en la calidad de la investigación que permiten la conformación de grandes bases de datos que amplían la capacidad de contrastación y generalización y la posibilidad de replicabilidad, esencial en algunas disciplinas; $\mathbf{x}$ ) previo a todo lo anterior, es necesario identificar que el XMLJATS es un medio y no un fin; asimismo, que no es un formato de lectura.

El formato XML-JATS implica: i) etiquetar los metadatos de identificación de un artículo científico (front); el texto completo (body), incluyendo las tablas, gráficas y ecuaciones (éstas últimas con MathML; así como las referencias bibliográficas (back) y los suplementos. ii) El XML-JATS se define por el marcaje de las etiquetas descritas y no por tener una extensión .xml. iii) La generación de archivos XML-JATS exige un sistema o herramienta de marcación; para este fin Redalyc pone a disposición Marcalyc, un desarrollo tecnológico que permite obtener archivos XML-JATS que cumplen con el estándar internacional. Marcalyc es un sistema web que garantiza que los usuarios trabajen con el sistema más actualizado y está diseñado para usuarios no informáticos que tengan experiencia básica en tratamiento de textos científicos.

La transición a la vía digital es irreversible. La mayoría de los usuarios (autores, lectores y público en general) acceden a la revista en su versión electrónica y al encontrarse con un PDF las ventajas tecnológicas que pueden adquirirse con la tecnología digital expuesta no se aprovechan.

Este es el contexto, no obstante, permanecen puntos de tensión. Si bien la visibilidad es una cuestión ésta resuelta si se trabaja con tecnología de punta: A) Existe una brecha tecnológica: es común encontrar que los portales de instituciones editoras de revistas y los de las propias revistas -salvo escasas excepcionesno aprovechan todas las posibilidades de los desarrollos tecnológicos. B) La brecha tecnológica se expande: Redalyc presenta diversas ventajas y valores agregados: formatos de lectura, búsqueda avanzada, indicadores bibliométricos, protocolos de interoperabilidad, etc., mientras los portales de revista han reducido sus servicios, en muchos casos sólo al PDF, etc. Bajo este contexto, parece un reto la transición digital rápida y extendida a las revistas regionales. Lo expresado pareciera indicarnos que el modelo debe cambiar en aspectos centrales.

Otro problema derivado de lo digital, es que las revistas adoptan procesos tecnológicos digitales, pero los procesos editoriales continúan sin cambio y sin adaptarse a las potencialidades de lo digital, lo que obedece -como se expresó- a la subordinación de lo digital a lo impreso.

Entre las limitaciones a superar se encuentran:

A) El tiempo de publicación: se continúa la subordinación a una periodicidad innecesaria en el ámbito digital, lo cual puede superarse mediante la publicación continua-periódica, que inicia y termina en función de la periodicidad definida, en la medida en que superen el proceso de dictamen; o mediante el cambio a publicación continua-permanente, que implica poner a disposición los artículos en la medida en que se postulen y superen el dictamen. Por otra parte, se termina con la problemática que representan los límites de cantidad de artículos por número, secciones fijas con dificultad de mantenerlas, etc.

B) La paginación continua del número, en algunos casos del volumen, que es totalmente innecesaria cuando lo que circula y se distribuye son los artículos más que la revista completa.

C) La extensión a 20-25 hojas, determinadas por las posibilidades de impresión de un fascículo.

Es importante destacar que parte de los obstáculos expuestos para la transición obedecen a que los sistemas tecnológicos exigen permanente actualización y son costosos. 
Con lo expuesto se concluye que para poder reunir las capacidades que permitan una transición tecnológica continua, es necesario cambiar el modelo operante por uno que permita a los editores de revistas de Acceso Abierto del Sur contar con las capacidades tecnológicas para poder competir con sus similares en el mundo. El resto de la encrucijada es responder cómo puede el editor integrar los procesos tecnológicos que le darán más elementos en un mundo altamente competitivo en la transmisión de mensajes.

\section{EL ACCESO ABIERTO Y LA SUSTENTABILIDAD}

El acceso abierto plantea, de una u otra forma, que el producto final de la investigación esté disponible sin costo para el usuario, de forma abierta y pública. En Latinoamérica, los proyectos, aunque colaborativos, fueron financiados por las universidades o por los consejos de ciencia y tecnología. Sin embargo, cabe destacar que:

- Los proyectos de AA surgieron por visiones locales y fueron expandiéndose y, en mayor o menor medida, legitimándose, pero no surgieron de visiones regionales de gran alcance.

- Al tiempo, los editores y las instituciones fueron destinando -en mayor o menor medida- recursos para pagar diversos servicios que les permitieran realizar sus tareas: hosting, dominios, DOI, gestores editoriales, marcaje de XML, asesoría de consultores, instalación o actualización de OJS, software de edición, etc.

- Los organismos responsables de impulsar las políticas científicas en diversos niveles no modificaron la estructura presupuestal. Continuaron las demandas de acceso a bases de datos y libros para las bibliotecas y, sólo en pocos casos, se destinaron presupuestos específicos para el acceso abierto; de cualquier manera, se trató de recursos escasos.

- La interoperabilidad y presencia de las revistas en diversos lugares implica tiempo y recursos que se acrecientan. Este proceso, la mayor parte de las veces, se realiza con recursos insuficientes.

- Las diferentes iniciativas, proyectos y plataformas regionales no han logrado conformar alianzas o soluciones integradas que permitan potenciar la ciencia de la región de manera colectiva.

Lo anterior obliga a replantear el modelo desde diversos enfoques, con el fin de garantizar la sustentabilidad del ecosistema de acceso abierto y público de Latinoamérica. Se pueden plantear los siguientes ejes de discusión:

A) La construcción de un modelo de conocimiento abierto y público, que implique mayores dimensiones que el acceso abierto y público de las revistas. Es necesario pasar a una política integral de AA, aunque los problemas que enfrentan las revistas obstaculizan la posibilidad de pensar en una construcción de dicha política sin tensiones.

B) La necesidad de integrar una estrategia para la construcción de infraestructura regional, o al menos institucional, para fortalecer y garantizar el acceso abierto y público. Las propuestas institucionales carecen de envergadura y capacidad de impactar al país y la región.

C) La necesidad de asumir colectiva, institucional y regionalmente el apoyo, financiamiento y desarrollo de los sistemas tecnológicos que sirvan a los intereses regionales, como el sistema OJS o Redalyc.

El Open Journal System es un claro ejemplo de un proyecto exitoso en su adopción, así como en los beneficios que ha dejado al fortalecimiento editorial de la región. Los beneficios son indiscutibles, así como la vocación de los impulsores. Sin embargo, también se ha mostrado una falta de conformación de una comunidad de desarrolladores, o de apropiación de dicha plataforma a las necesidades particulares de instituciones o revistas con una perspectiva de largo aliento en la región. Las revistas la usan en su versión por defecto, dejando fuera todas las potencialidades del código abierto. Destacan las decenas o cientos 
de iniciativas personales, de grupos de académicos o de instituciones para apoyar su implementación y capacitación, donde sobresale la falta de una coordinación y corresponsabilidad regional en un sistema que ha propiciado beneficios incuestionables, pero cuya implementación depende de la capacidad de gestión, tecnológica, del tiempo, etc.

Siempre se pensó en intensificar el AA, pero no se pensó en la sostenibilidad financiera. Construir una infraestructura tecnológica de AA resulta inaplazable, de forma que la academia retome el control del proceso editorial y se apoye en los procesos tecnológicos ofertados por terceros.

\section{REDALYC SE RECONFIGURA}

A 15 años de su creación, es menester un ejercicio de reconocimiento y agradecimiento.

Gracias a la Universidad Autónoma del Estado de México por apoyar desde su creación esta iniciativa; por hacerla posible y ser el soporte financiero desde su inicio hasta la actualidad. Al Cuerpo Académico de Difusión y Divulgación de la Ciencia, por acogerla y respaldarla.

Gracias a todos los aliados, individuales e institucionales, colegas que nos han acompañado y compartido. Nombrar sería excluir, gracias a todos. Gracias a todos los editores iberoamericanos, que con su escrupuloso trabajo, constituyen la legitimidad de nuestro quehacer y el suyo. Gracias a los más de 100 millones de usuarios anuales, que confían en la calidad de los contenidos de esta ventana de conocimiento. Gracias especiales al equipo de Redalyc, que construye día a día, con convicción y entusiasmo, esta posibilidad de acceso al conocimiento, sin restricciones.

Redalyc comenzó en 2003 con el objetivo principal de contribuir a la visibilidad de las revistas científicas publicadas en la región, en un tiempo en el cual la mayoría de ellas no tenía presencia en la Web. Hoy, la colección de Redalyc contiene más de seiscientos mil artículos a texto completo de cerca de 1.300 revistas con revisión por pares, de Acceso Abierto, publicadas por 622 editoriales de 22 países de América Latina, España y Portugal.

Redalyc ha respondido con creces a las necesidades de visibilidad, posicionamiento y consolidación de la literatura científica regional.

Desde el inicio hasta la fecha, hemos ofrecido profesionalización, interoperabilidad, presencia, tráfico, internacionalización, indicadores de producción y consumo, identificación de autores y más recientemente, escalar a la tecnología XML-JATS llevando a las publicaciones científicas a la nueva era digital de la sociedad del conocimiento.

La presencia en internet y muchos otros aspectos ya no son el desafío. Hoy el Acceso Abierto, y por ende Redalyc, enfrentan otros retos. El escenario en torno a la comunicación científica ha cambiado.

El Acceso Abierto ha tomado formas diversas en cada región, es interpretado, retomado y modificado de maneras muy distintas. Las empresas editoriales han convertido el acceso abierto en un nuevo modelo de negocio mediante los pagos por publicar, imposible de sostener en nuestra región.

Los sistemas de evaluación nacionales han adoptado las métricas de la llamada ciencia de corriente principal, lo que pone en entredicho la articulación de la ciencia con la sociedad.

Existe el creciente control del circuito de la comunicación científica por las grandes editoriales comerciales, poniendo en riesgo el futuro de la publicación académica.

\section{CONSIDERACIONES FINALES}

Bajo los contextos y tensiones mencionados, nos gustaría emitir unas consideraciones a manera de reflexiones finales. 
El sentido -teleológico- del acceso abierto es difícilmente cuestionable desde una perspectiva global y de beneficios para el desarrollo de la ciencia y la humanidad. Sólo se oponen los que se benefician de una u otra forma de cerrar el conocimiento bajo muros de pago.

La autonomía de universidades y países es claramente puesta en duda por el control del circuito de la ciencia que han adquirido las empresas en los últimos años. No sólo es una cuestión de recursos económicos, tal control pone en entredicho la autonomía de las políticas de ciencia y tecnología de los países y la contribución al desarrollo, articulación y problemáticas del cada país.

Los sistemas de evaluación -cada vez más criticados por los distintos actores que intervienen en la investigación-, de no modificarse, terminarán haciendo fracasar el acceso abierto o ajustándolo a sus fines, al mantener los APC -lógica comercial- pero permitir el acceso.

La falta de participación y apoyo en la sustentabilidad financiera de un proyecto que beneficia a toda la región pone en entredicho proyectos como Redalyc, que nacen financiando -de manera centralizada- la visibilidad y posicionamiento de la producción científica regional.

Redalyc no impulsará de forma significativa desarrollos nuevos que incrementen la visibilidad regional, mientras no cambie el entorno, mantendrá los existentes en la medida de sus posibilidades financieras. Como proyecto académico, se dirigirá en los siguientes años a apoyar con la tecnología desarrollada proyectos cooperativos financiados y sostenidos por la comunidad. Como proyecto de innovación y desarrollo tecnológico mostrará una plataforma de publicación basada en los mayores estándares internacionales, pero que no dependerá de ningún servicio centralizado para su operación que pueda poner en entredicho su objetivo inicial: Redalyc2020.

Se retoma el principio de Tim Berners-Lee de que nadie puede controlar y adueñarse de la red. Parafraseando a Berners-Lee podemos decir que en el ámbito internacional lo que en un tiempo fue una rica y variada selección de revistas científicas auspiciadas por la academia, ha dado paso a la concentración de revistas y servicios por parte de unas pocas y muy poderosas empresas que están muy alejadas del interés de compartir por el simple hecho de beneficiar a la humanidad y disminuir la exclusión. Latinoamérica se resiste impulsando un acceso abierto no comercial, cooperativo, sustentable y protegido. Debemos trabajar conjuntamente para mantener los proyectos de beneficio regional.

Redalyc2020 avanzará a la web semántica de la publicación científica, insertando metadatos semánticos y ontológicos para ampliar y reforzar la interoperabilidad de esos agentes inteligentes - máquinas-en beneficio de sus operadores y creadores: la sociedad.

\section{Notas}

1 Presentación invitada en el $3^{\circ}$ Congreso Internacional de Editores Redalyc "Construyendo el modelo de publicación académica del sur global”. Trujillo, Perú, 16-18 mayo 2018. http://congreso.redalyc.org/ocs/public/congresoEditores/ index.html

2 Declaración de la Primera Reunión de Consorcios de Iberoamérica y el Caribe (http://reuniondeconsorcios.conricyt.mx/wp-content/uploads/2017/09/OK-DECLARACIO\%CC\%81NDE-LA-REUNIO\%CC\%81N-DE-CONSORCIOS-DE-IBEROAME\%CC\%81RICA-Y-EL-CARIBE-1-1.pdf). Ver Plan S y AmeliCA (http://www.amelica.org/index.php/2019/01/10/amelica-vs-plan-s-mismo-objetivo-dos-estra tegias-distintas-para-lograr-el-acceso-abierto/)

3 Declaración México (http://www.accesoabiertoalyc.org/declaracion-mexico/) 